The Journal of Neuroscience 2012; 32(16):5585-5597

\title{
Preclinical retinal neurodegeneration in a model of multiple sclerosis
}

\author{
Richard Fairless, Sarah K. Williams, Dorit B. Hoffmann, Aleksandar Stojic, Sonja Hochmeister, \\ Frank Schmitz, Maria K. Storch and Ricarda Diem
}

Departments of Neurology and Anatomy and Cell Biology, University of the Saarland, Homburg/Saar, Germany; Department of Neuro-Oncology, University Clinic Heidelberg, Heidelberg, Germany and Department of Neurology, Medical University of Graz, Graz, Austria

Correspondence : Dr. Ricarda Diem, Department of Neuro-Oncology, University Clinic Heidelberg, Im Neuenheimer Feld, Heidelberg, Germany; E-mail: ricarda.diem@med.uni-heidelberg.de

\author{
Deepali Mathur ${ }^{1}$, Alberto Hernandez Cano ${ }^{2}$ \\ ${ }^{1}$ University of Valencia, Valencia, Spain; ${ }^{2}$ Prince Felipe Research Center, CIPF, Valencia, Spain
}

\begin{abstract}
Background
Multiple Sclerosis (MS) is a chronic inflammatory disease that affects the central nervous system (CNS) and causes demyelination and neuronal loss, leading to axonal dysfunction. Over the past decade there has been a considerable amount of discussion about whether immune-mediated myelin damage is a primary or secondary event for axon degeneration in MS. Previous investigations have demonstrated that axonal loss is a consequence of inflammatory demyelination in neurodegenerative diseases. In an early relapsing remitting phase of MS, the disease process targets myelin, which is followed by axonal transection in a secondary progressive stage that causes irreversible neurological disability. However, recent in vivo imaging studies using optical coherence tomography (OCT) have shown that neurons in the retina that lack myelin sheath degenerate as well, suggesting that neurodegeneration ensues in demyelinated areas of the CNS. ${ }^{1}$ Reduced thickness of the retinal layer as observed by OCT in MS patients affected with optic neuritis further support the notion that damaged neurons in the retina contribute to the functional deficits in MS. ${ }^{2}$ Optic neuritis is an inflammatory condition of the optic nerve that undergoes demyelination and neurodegeneration.
\end{abstract}

Animal models of experimental autoimmune encephalomyelitis (EAE) are very useful for studying the pathogenesis of MS. Because the disease course and clinical symptoms of MS patients are distinct, many well-characterized EAE models have been established. Induction of EAE often results in the development of optic neuritis. One study showed that when animals were sensitized with proteolipid protein, optic neuritis developed by day 17 post-immunization (p.i.). ${ }^{3}$ In another model, immunization of animals with myelin oligodendrocyte glycoprotein (MOG) produced inflammation of the optic nerve. ${ }^{4}$ In this report, Fairless et al. assessed the relationship between immune response and neurodegeneration in a rat model before observable symptoms of optic neuritis developed.

\section{Study Design}

To induce EAE, the authors immunized the animals with MOG, which resulted in clinical symptoms of both MS and optic neuritis. Retinal ganglion cells (RGCs) were quantified at different time points during the induction phase of the disease. In order to obtain an exact RGC count, axotomy of the optic nerve was performed, which prevented contamination of RGC with other non-neuronal cells. Fluorescence microscopy revealed a significant reduction in RGC count as early as day 5 p.i. In addition, visualization of apoptotic cells in the ganglion cell layer on day 5 p.i. further supported the hypothesis that ganglion cells degenerate before the onset of the disease. Together, these data suggested that neurodegenerative events precede the inflammatory processes.

During the preclinical phase of the disease, histopathological analysis did not detect axon degeneration, beta-amyloid precursor protein ( $\beta$-APP) accumulation, or demyelination of the optic nerves. However, at the ultrastructural level, axon density was reported to be compromised on day 7 p.i. Moreover, retracted axons with surplus vacuoles were observed before the manifestation of the disease. It is intriguing that intact myelin surrounded transected axons, which suggests that demyelination might be the consequence of axon degeneration. To evaluate the cellular arm of adaptive immunity, immunohistochemistry of CD 3 positive T cells was performed. The results showed that these cells do not infiltrate into the CNS parenchyma until disease onset. Absense of infiltrated immune cells in central nervous system during the induction phase suggested inflammation to be a secondary event to neurodegeneration. However, some of these inflammatory infiltrates were visualized in the optic nerve meninges and choroid tissue. Since these meningeal T cells correlate with the numbers of activated microglia, the authors suggest their role in stimulating the innate immunity. In contrast the humoral arm of adaptive immunity was assessed by measuring antibodies directed against myelin derived protein in blood and optic nerve tissue. Significant levels of antibodies were found on day 10 p.i. in the optic nerve head indicating that the blood brain barrier (BBB) was incomplete in this area (Fairless et al., 2012). Absence of T cell infiltrates and antibodies in the central nervous system indicated that the BBB integrity was not compromised during the induction phase of the disease. One should mention here that other than $T$ cells and $B$ cells producing antibodies, evaluation of complement proteins activated as a result of neuronal injury would have revealed additional insight into disease pathology. In secondary progressive MS patients, the opsonin C3d was shown to be deposited in demyelinated areas that provided evidence for its contribution to disease progression as well as acute inflamma- 
tion. ${ }^{5}$ In addition activated complement protein products such as C9neo and C3d too are found exclusively in areas of active demyelination and MS brains. ${ }^{6}$

The authors in this report showed that BBB integrity was not disrupted until the disease onset but increased with disease progression. To examine the integrity of the BBB further, the authors studied albumin leakage and the expression of astrocyte marker and tight junction protein that form part of the barrier. Albumin extravasation into the central nervous system was not apparent in the induction phase. However, the astrocyte marker such as aquaporin 4 showed an irregular staining on day 10 p.i. which may be the result of dysregulated expression of occludin, a tight junction protein on day 10 p.i. Blood retinal barrier (BRB) integrity on the other hand was disrupted during the preclinical phase of the disease. This is supported by the findings that small amounts of albumin diffused through the barrier as early as day 5 p.i. and was observed in larger amounts as the preclinical phase continued. As early as day 5 p.i. astrocyte marker glial fibrillary acidic protein (GFAP) was expressed in significantly higher amounts indicating astrocyte reactivity. Surprisingly, the authors did not determine GFAP expression while assessing BBB integrity.

On day 5 p.i. the authors observed a significantly elevated expression of CD 68 marker on activated microglia and macrophages suggesting that resident cells of the central nervous system were activated along with RGC degeneration during the induction phase. In summary, Fairless et al. 'using an animal model of MS, show that RGC bodies degenerate early in the course of the disease preceding other events such as demyelination and inflammation. At the ultrastructural level, signs of axon degeneration were visualized in the retina when significant number of RGC bodies were found to have declined. These findings suggest that the trigger that likely initiates the disease process appears to develop within the retina. In addition, myelin destruction as a result of immune cell infiltration into the central nervous system was evident at a much later stage. The resident cells of the central nervous system comprising of microglia and macrophages were found activated in the preclinical phase of the disease. It should be noted here that small numbers of meningeal $T$ cell infiltrates observed in early period might be responsible for stimulating the resident immune cells and subsequently targeting ganglion cell bodies and their axons in the retina. Hence, the mechanism that promotes neurodegeneration may be due to the activation of either the innate immunity or adaptive immunity.

\section{Implications}

In conclusion, it remains elusive whether MS should be considered primarily a demyelinating or neurodegenerative disease. Although there is a strong body of evidence suggesting demyelination being the primary contributor to pathogenesis of MS because many recent studies have reported neuronal loss in myelin deficient retina. The findings of this study describe a critical role of innate immunity mediating the process of neurodegeneration in the preclinical phase of the disease. This led the authors to conclude that early inflammatory changes may begin the process of neurodegeneration in the retina before major clinical symptoms of the disease exacerbates. Further investigation confirming and extending the concept of innate immunity as the primary driving force is warranted.

\section{Acknowledgements}

We would like to thank Dr. Martina Kirstein for reviewing the manuscript and Dr. Fernando Martinez-Garcia for support.

doi : 10.5214/ans.0972.7531.190307

\section{References}

1. Saidha S, Syc SB, Ibrahim MA et al. Primary retinal pathology in multiple sclerosis as detected by optical coherence tomography. Brain 2011; 134: $518-533$.

2. Tátrai $\mathrm{E}$, Simó $\mathrm{M}$, Iljicsov $\mathrm{A}$, et al. In Vivo Evaluation of Retinal Neurodegeneration in Patients with Multiple Sclerosis. PLoS ONE 2012; 7(1): e30922

3. Potter NT and Bigazzi PE. Acute optic neuritis associated with immunization with the CNS myelin proteolipid protein. Investigative Ophthalmology and Visual Science 1992; 33(5): 1717-1722.

4. Storch MK, Stefferl A, Brehm U et al. Autoimmunity to myelin oligodendrocyte glycoprotein in rats mimics the spectrum of multiple sclerosis pathology. Brain Pathol 1998; 8: 681-694.

5. Ingram G, Hakobyan S, Robertson NP, et al. Complement in multiple sclerosis: its role in disease and potential as a biomarker. Clin Exp Immunol. 2009; 155: 128-139.

6. Barnett $\mathrm{MH}$ and Prineas JW. Relapsing and remitting multiple sclerosis: pathology of the newly forming lesion. Ann Neurol 2004; 55: 458-468. 